\title{
PAPER \\ Fast S-Parameter Calculation Technique for Multi-Antenna System Using Temporal-Spectral Orthogonality for FDTD Method
}

\author{
Mitsuharu OBARA $^{\dagger \text { a) }}$, Student Member, Naoki HONMA ${ }^{\dagger}$, Member, and Yuto SUZUKI ${ }^{\dagger}$, Student Member
}

\begin{abstract}
SUMMARY This paper proposes an S-parameter analysis method that uses simultaneous excitation for multi-antenna systems. In this method, OFDM (Orthogonal Frequency Division Multiplexing) and CI (Carrier Interferometry) pulse generation schemes are employed for maintaining the orthogonality among the excited signals. In OFDM excitation schemes, the characteristics of the neighboring antennas can be calculated by assigning different frequency subcarriers exclusively. CI enables the simultaneous verification of the antennas distant enough since this method can provide temporal orthogonality. Combining these two methods yields the simultaneous analyses of array antennas with both narrow and wide element spacing. The simulation of a $2 \times 2$ multi-antenna shows that the results of the proposed method agree well with those of the conventional method even though its computation speed is more 4 times that of the conventional method.

key words: FDTD, OFDM, CI, S-parameter, multi-antenna
\end{abstract}

\section{Introduction}

Recently, Multiple-Input Multiple-Output (MIMO) systems [1] and diversity antenna [2] are widely used in many areas of wireless communication. Diversity antennas are also frequently used to counter multipath fading. To design a diversity antenna with the desired characteristics, an efficient simulation method is needed. The FDTD (Finite-Difference Time-Domain) method [3] is convenient for analyzing complicated structures including the antennas and propagation environments. However, computation time rapidly becomes excessive since it increases in proportion to the number of antennas, since each antenna requires individual simulation. For example, MIMO base stations have many antennas, and the surrounding environments, such as the antenna tower structure, and adjacent base stations, and so on, must be taken into account. Therefore, a fast FDTD analysis for multi-antenna systems is required.

In this paper, a fast FDTD analysis method that uses simultaneous excitation is proposed for multi-antenna systems. In this method, OFDM (Orthogonal Frequency Division Multiplexing) pulse and CI (Carrier Interferometry) [4] are employed to maintain the spectrum and time orthogonality among the excited signals. The spectrum orthogonality is used for calculating the characteristics of the neighboring antennas by assigning different OFDM subcarriers exclusively. CI enables the simultaneous verification of antennas that are distant enough by controlling the power envelope of

Manuscript received June 10, 2011.

Manuscript revised November 16, 2011.

${ }^{\dagger}$ The authors are with the Graduate School of Engineering, Iwate University, Morioka-shi, 020-8551 Japan.

a) E-mail: t2310007@iwate-u.ac.jp

DOI: 10.1587/transcom.E95.B.1338 the pulse in the time domain. These methods can achieve much faster analysis than the FDTD method with the conventional pulse. Section 2 details the concept of pulse generation and the analysis method. Section 3 shows the numerical analysis results, and clarifies the effectiveness of the proposed method is clarfied.

\section{Basic Concept}

\subsection{Orthogonality Using Frequencies}

The OFDM system divides the available frequency band into narrow subcarriers and multiplexes them orthogonally. The frequency interval, $\Delta f$, is given by

$$
\Delta f=1 / T
$$

where, $T$ is signal length. If the frequency interval does not meet this condition, the subcarrier interference is not insignificant. Figure 1 shows the conceptual sketch of the signal arrangement in the frequency domain. The antennas can be analyzed by assigning signals to subcarriers alternately. The signal numbers, $\sharp 1$ and $\sharp 2$ in Fig. 1 correspond to the antenna numbers, $\sharp 1$ and $\sharp 2$. The signal transformation is given by

$$
S_{i}(t)=\sum_{n=0}^{\frac{N-1}{k}} \mathfrak{R}\left\{z_{(i-1)+n k} \exp \left(j 2 \pi f_{(i-1)+n k} t\right)\right\}
$$

where $N$ is the number of the subcarriers, $n$ is the subcarrier index number, \# $i$ is the signal/antenna number, $S_{i}$ represents signals in the time domain, $k$ is the number of antennas, $z_{(i-1)+n k}$ represents the signals in the frequency domain,

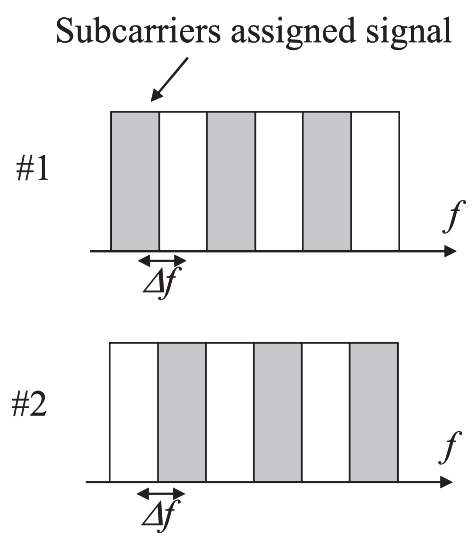

Fig. 1 Signal arrangement. 
$f_{(i-1)+n k}$ is the center frequency of the subcarriers and $\mathfrak{R}\{\cdot\}$ means the real part of the complex number. Additionally, transmitting the signals repeatedly can reduce the interference among the signals, since frequency orthogonality can be maintained. In this paper, this method is used to obtain the characteristics of the antennas that are placed close together.

\subsection{Temporal Orthogonality}

Analysis using CI pulse can keep the orthogonality by exploiting the propagation time. In CI, the same amplitude value is assigned to all subcarriers being analyzed. CI signals can be expressed as

$$
z_{l}=e^{-j 2 \pi \frac{N_{t}(l-1)}{N}}
$$

where, $z_{l}$ is the signals in frequency domain, $l$ is the index number of subcarrier, $N$ is the number of the subcarriers, and $N_{t}$ is linear phase offset in time domain. The frequency domain signals shown in (3) are transformed into the time domain signals by (2). The CI method can collect most of the signal powers in a short period of time.

Figure 2 shows the concept of analysis based on the CI pulse. In this method, the antennas are separated by sufficient distance and excited together. The incident and arrived signals can be separated by using the temporal orthogonality thanks to the propagation time.

This paper uses this method to obtain the characteristics between the antennas that are sufficiently separated. The analysis that uses the CI technique exploits temporal orthogonality only to avoid the interference among pulses. Therefore, this method does not suffer from degradation in spectral resolution. When $2 \times 2$ antennas are simulated by the OFDM technique, the spectral resolution becomes $1 / 2$ that of $1 \times 1$ antennas. In order to obtain same resolution with only the OFDM technique, the time-step number in FDTD

(a)
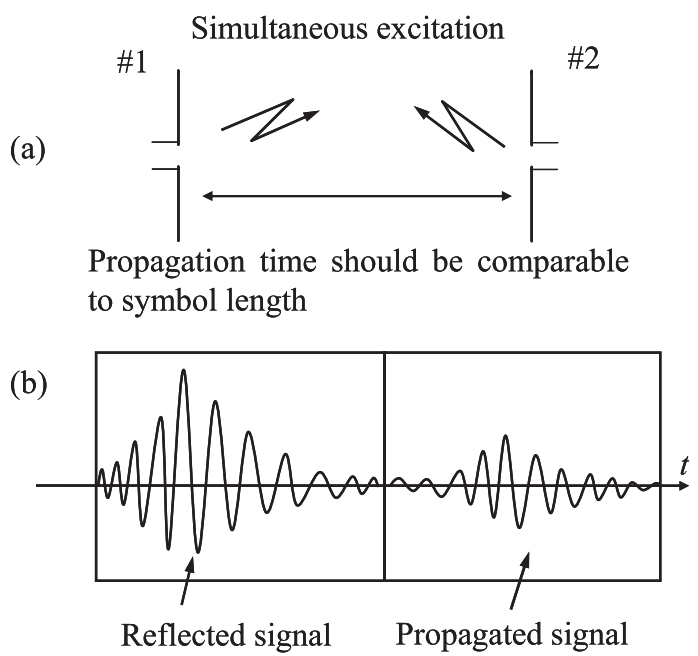

Fig. 2 Sketch of the antennas and signals in the analysis that uses the CI method: (a) conceptual sketch of the antennas, (b) observed time domain signal. must be raised. On the other hand, CI suits the situation where the scattering parameter strongly depends on the frequency. For example, CI can be used for antennas that have very narrow bandwidth. Therefore, either the OFDM or the CI technique can be used depending on simulation model and requirements.

\subsection{Scattering Parameter Analysis}

This section describes a method to calculate the scattering parameter in the FDTD method. In the FDTD method, the total voltage and current in the time domain can be calculated first. After that, the voltage and current waveform in the time domain are transformed into frequency domain values by Fourier transformation. The scattering parameter is obtained from the voltage and current in the frequency domain. The complex envelopes of inward and outward propagating waves in the frequency domain are expressed as,

$$
\begin{aligned}
& \boldsymbol{a}=\frac{1}{2}\left(\boldsymbol{v} / Z_{0}{ }^{1 / 2}+i Z_{0}{ }^{1 / 2}\right) \\
& \boldsymbol{b}=\frac{1}{2}\left(\boldsymbol{v} / Z_{0}^{1 / 2}-i Z_{0}^{1 / 2}\right),
\end{aligned}
$$

$Z_{0}$ is the reference impedance to define the scattering parameters, and $\boldsymbol{v}$ and $\boldsymbol{i}$ denote the voltage and current vector in the frequency domain, respectively. The relationship among the scattering parameters, $\boldsymbol{a}, \boldsymbol{b}$ is expressed as

$$
\boldsymbol{b}=\boldsymbol{S a}
$$

where $S$ is the scattering parameter matrix [5]. The scattering parameter is obtained as

$$
S_{k j}=b_{k} / a_{j}
$$

where, $j$ and $k$ are the port numbers of antennas. $S_{k j}$ is the $k$-th line and $j$-th column component of $\boldsymbol{S}$. To calculate all components of $S$, only one port out of all ports should be excited in the conventional method. Therefore, individual analyses for all ports are required.

\section{Numerical Results}

\subsection{Analysis Condition}

This section analyzes the scattering parameters of $1 \times 1$ or $2 \times 2$ biconical antennas. Figure 3 shows the analysis models. Figure 3(a) is the model for the analyses using OFDM or CI technique, where antenna element spacing is $D$. The dimensions of the analysis region are $(D+0.68) \mathrm{m} \times 0.44 \mathrm{~m}$ $\times 0.305 \mathrm{~m}$. Figure 3 (b) shows the model for the combined OFDM and CI technique, where the two arrays comprising two close antennas are located at the ends of the analysis region. The dimensions of the analysis region are $(D+0.68) \mathrm{m}$ $\times 0.78 \mathrm{~m} \times 0.305 \mathrm{~m}$. The length of the biconical antennas is $0.105 \mathrm{~m}$ and two or four antennas are arranged symmetrically in the analysis region. $N_{0}$ is defined as the number of time steps corresponding to the symbol length, and 


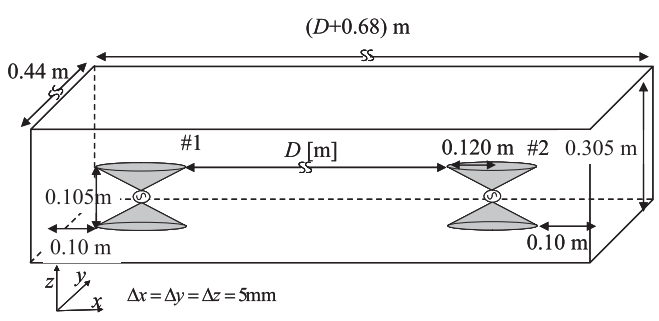

(a) For OFDM or CI technique

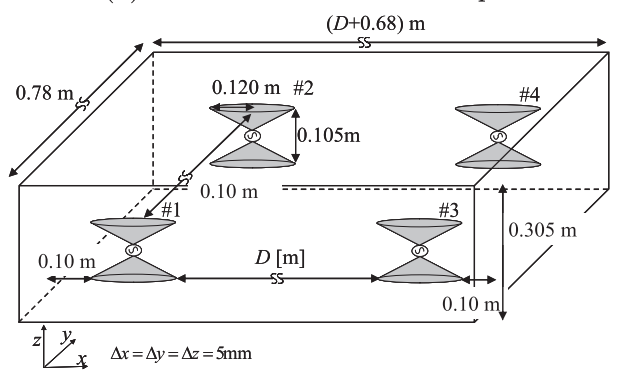

(b) For both OFDM and CI technique

Fig. 3 Analysis model.

Table 1 Parameters for analysis.

\begin{tabular}{|c|c|}
\hline Antenna & Biconical antenna \\
\hline Feeding model & Delta-gap feed \\
\hline Cell size & $5 \mathrm{~mm}$ \\
\hline Absorbing B.C. & PML \\
\hline Number of PML layer & 4 \\
\hline Analysis frequency & $0 \sim 5 \mathrm{GHz}$ \\
\hline Time step & $9.1477 \mathrm{ps}$ \\
\hline
\end{tabular}

$N_{0}=1024$ in this simulation. Therefore, the propagation distance during one symbol signaling is expressed as, $L=c\left(N_{0} \Delta t\right)$, where $c$ is the wave velocity in a vacuum, $\Delta t$ is the time step. In this paper, the propagation distance during one symbol signaling $L$ is $2.810 \mathrm{~m}$. Details of the FDTD analysis configurations are summarized in Table 1.

In the proposed method, the total number of subcarriers, $N$, is 1024 , the frequency spacing, $\Delta f$, is $0.106 \mathrm{GHz}$. For comparison, the Gaussian pulse is used and two or four individual analyses are performed in total. $N_{1}$ is the number of the necessary time steps; it depends on the separation distance of the two facing arrays, i.e.,

$$
N_{1}=(D+L) / c \Delta t .
$$

In the following discussion, the ideal results obtained by the Gaussian pulse excitation are called the conventional method.

\subsection{Analysis Using OFDM Technique}

Figure 4 shows OFDM pulse waveforms. Antenna $\sharp 1$ transmits signal 1 shown in Fig. 4(a), and antenna $\sharp 2$ transmits signal 2 shown in Fig. 4(b). In the proposed method, analyses that use OFDM pulses require $2 N_{0}$ time steps for excitation and analysis. This is because exciting an OFDM pulse repeatedly can reduce the intersymbol interference.

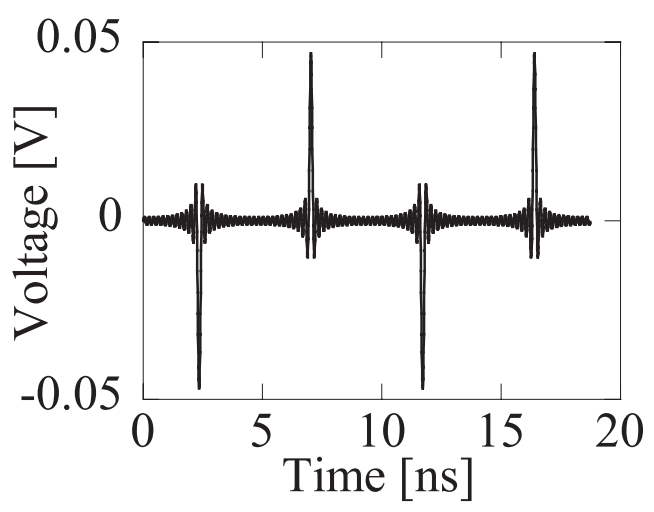

(a) Signal 1

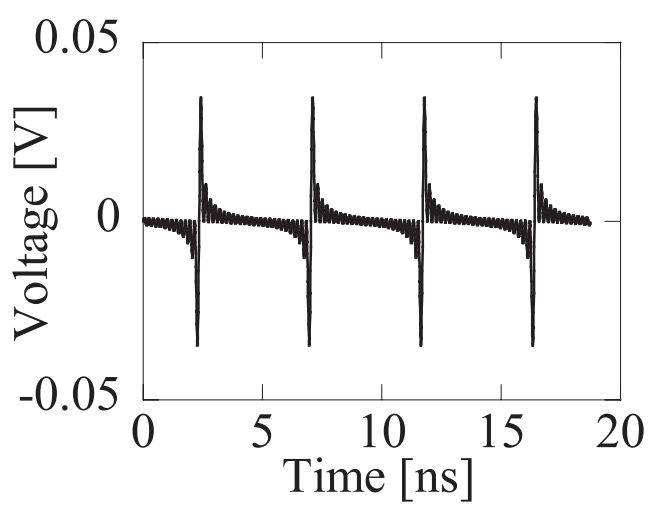

(b) Signal 2

Fig. 4 OFDM pulse waveforms.

To evaluate the inter-subcarrier interference of the proposed method, simulations with one and two excitations are carried out and compared. Similarly, the results of the conventional method that uses Gaussian pulse with $N_{0}$ and $2 N_{0}$ time steps are compared.

Figure 5 shows the RMS (Root Mean Square) error of the scattering parameters with one $\left(N_{0}\right)$ and two $\left(2 N_{0}\right)$ excitations. Here, the symbol length is set to $N_{0} \Delta t$. The antenna spacing, $D$, is changed from $0.01 \mathrm{~L}$ to $1.0 \mathrm{~L}$. The error is calculated by comparing to the result obtained from 4096 time steps analysis with Gaussian pulse. The results in Fig. 5 show that the longer propagation distance between the antennas becomes, the higher RMS errors of the scattering parameter become. This tendency is seen in both the conventional and proposed methods with $N_{0}$ time steps.

There are two reasons that cause this error. One is the propagation time between two antennas. When $D / L$ is higher than 0.7, the RMS errors in $S_{21}$ become greater than $30 \%$ when the conventional method with $N_{0}$ time steps is used. Since the transmitted signal from one antenna cannot reach another antenna, the $S_{21}$ cannot be calculated correctly. Another reason is the orthogonality among the subcarriers, and this problem happens only in the proposed method. The RMS errors of $S_{21}$ with $N_{0}$ time steps are greatly large compared with that with $2 N_{0}$ time steps in the proposed method. The length of the Fourier transform win- 


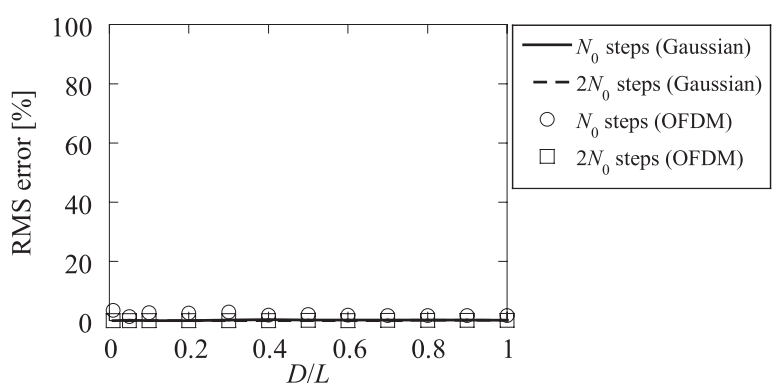

(a) $S_{11}$

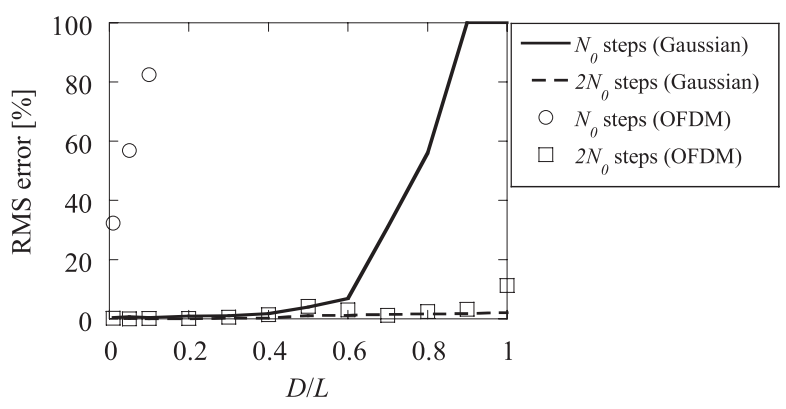

(b) $S_{21}$

Fig. 5 RMS error of scattering parameter versus antenna distance, $D$, in OFDM technique.

dow is $N_{0}$ time steps and the signal must be continuously observed during the window span. Since the lack of the signal in the time domain degrades frequency orthogonality, the RMS error is increased when the symbol is transmitted just once.

By contrast, the proposed method with $2 N_{0}$ time steps can calculate scattering parameter with the RMS errors less than about $10 \%$. In this case, the window is positioned at the last $N_{0}$ time steps, since higher accuracy is expected when the voltage and current with steadier state are used. It is found that this technique can improve the RMS error in $S_{21}$ by more than $30 \%$ compared with the proposed method with $N_{0}$ time steps. According to these results, the proposed method can calculate the scattering parameter of two antennas with half computation time of the conventional method in total.

Figure 6 shows the frequency characteristics of the scattering parameter at $D=0.05 L$. The scattering parameters are successfully obtained by the proposed method and agree very well with those of the conventional method in this simulation.

\subsection{Analysis Using CI Technique}

Figure 7 shows the CI pulse waveform. The antennas $\sharp 1$ and $\sharp 2$ transmit the same signal shown in Fig. 7. The linear offset $N_{t}$ is set to 21. As indicated by the Fig. 7, most of powers of CI pulse are collected in the short period of excitation pulse. After this, array spacing $D$ is changed from $0.1 \mathrm{~L}$ to $2.0 \mathrm{~L}$ by $0.1 L$ step. Figure 8 shows the RMS error of the scatter-

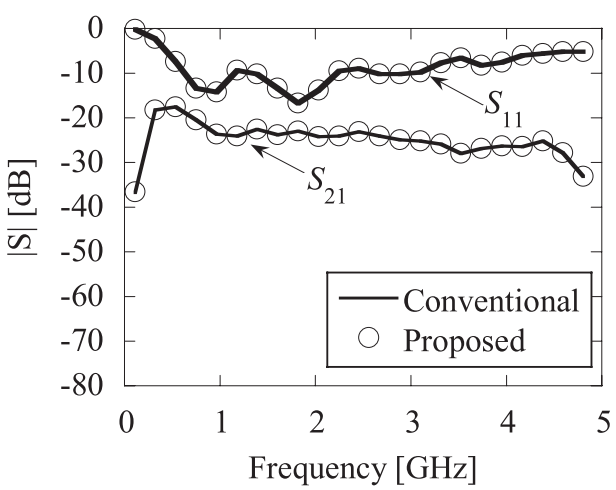

Fig. 6 Comparison of the simulated scattering parameters: conventional method and proposed method using OFDM technique $(D / L=0.05)$.

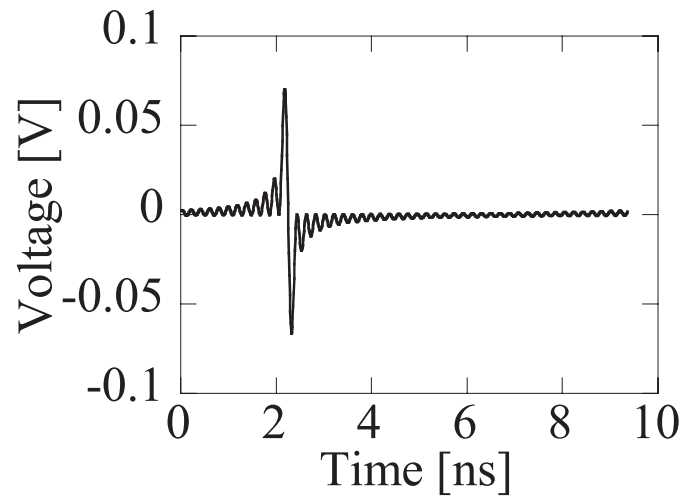

Fig. 7 CI pulse waveform.

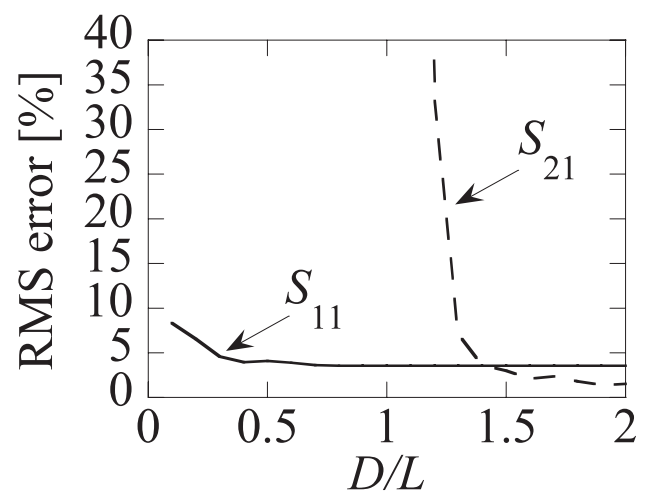

Fig. 8 RMS error between conventional method and proposed method using CI technique.

ing parameters of the proposed method compared with that of the conventional method. The results of Fig. 8 show that $S_{21}$ has large RMS errors if $D / L$ is lower than 1.5. This is because array spacing $D$ is not large enough to maintain orthogonality in the time domain. By comparing the results in Fig. 5 , it can be seen that the OFDM scheme with $2 N_{0}$ timesteps yields lower RMS error that the CI scheme. However, the spectral resolution of the OFDM scheme is half that of the CI scheme. This simulation assumed biconical antennas, which have broad bandwidth. That is, the scattering parameter variation in the frequency is not significant, and high 


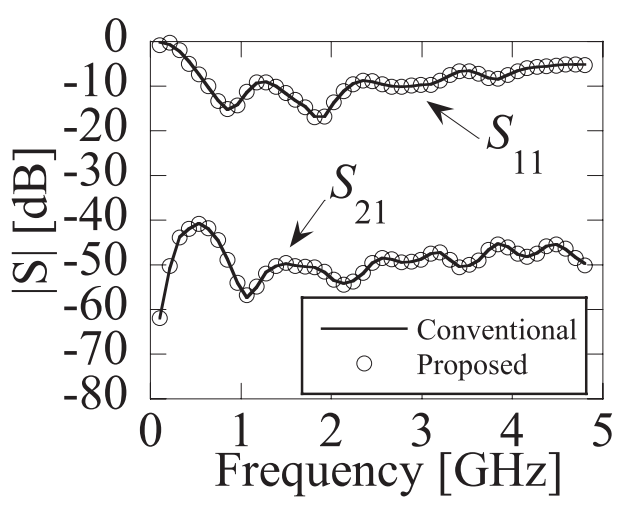

Fig. 9 Comparison of simulated scattering parameters between conventional method and proposed method using CI technique $(D / L=2.0)$.

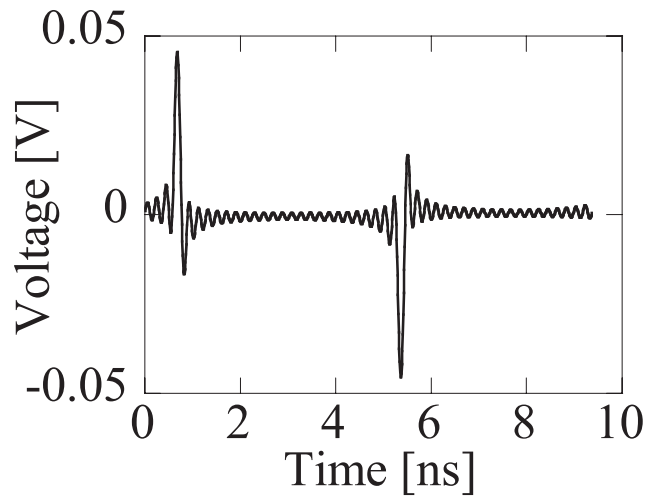

(a) Signal 1

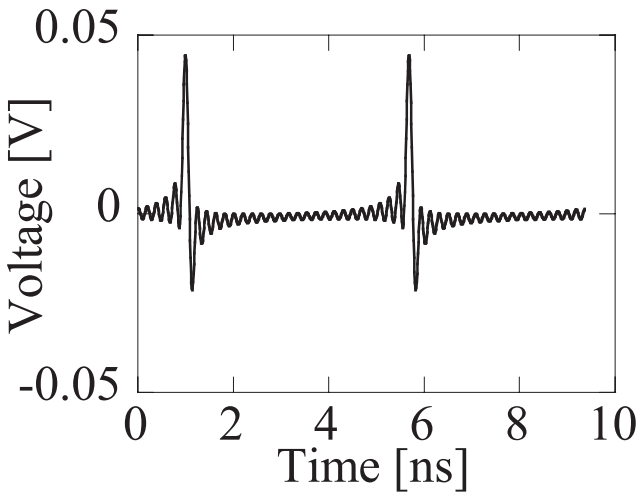

(b) Signal 2

Fig. 10 Waveforms using combined OFDM and CI technique.

spectral resolution is not required. When the antennas have narrow bandwidth, such as patch antennas and inverted-F antennas, the CI scheme would be used to evaluate the frequency characteristics of these antennas.

Figure 9 shows the scattering parameters using CI pulses with $D / L=2.0$. The scattering parameters obtained by the proposed method agree very well with that of the conventional method.

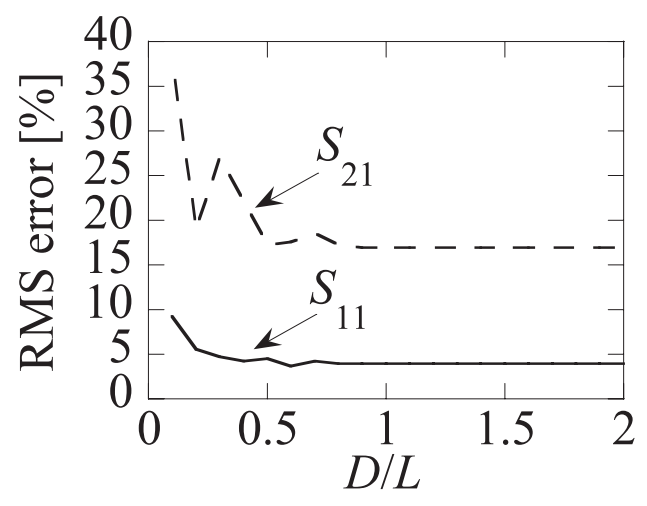

(a) $S_{11}, S_{21}$

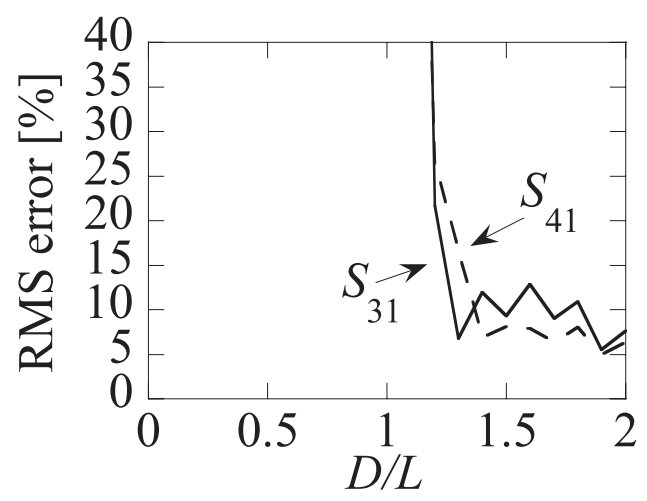

(b) $S_{31}, S_{41}$

Fig. 11 RMS error between conventional method and proposed method using combined OFDM and CI technique.

\subsection{Analysis Using Both OFDM and CI Technique}

In this scheme, the OFDM technique is used for the simultaneous analysis of the two neighboring antennas, and the CI technique is applied to the two distant arrays. However, CI technique can not be applied to the OFDM pulse without modification if the subcarriers are assigned alternately to two neighboring antennas. Therefore, the $\mathrm{CI}$ technique is applied to alternate subcarriers in this simulation. Figure 10 shows the waveforms of combined OFDM and CI method. Since a CI pulse is combined with an OFDM pulse the signal exhibits two peaks in each symbol period. In this simulation, antennas $\sharp 1, \sharp 3$ are excited by signal $\sharp 1$ shown in Fig. 10(a). Similarly, antennas $\sharp 2, \sharp 4$ are excited by signal $\sharp 2$ shown in Fig. 10(b). Figure 11 shows the RMS error of the scattering parameters of the proposed method using combined OFDM and $\mathrm{CI}$ technique compared with the conventional method. There are large RMS errors in $S_{31}$ and $S_{41}$ if $D / L$ is lower than 1.5. This is because array spacing $D$ is not enough to maintain orthogonality in the time domain.

In contrast, the results of $S_{21}$ have errors of around 10 to $20 \%$. This is caused by the degradation in frequency orthogonality. When the CI technique is used, the number of excitation symbols must be at least one since the CI tech- 


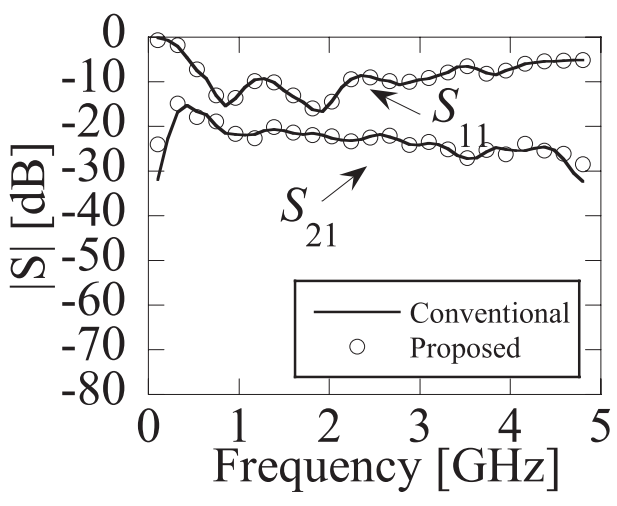

(a) $S_{11}, S_{21}$

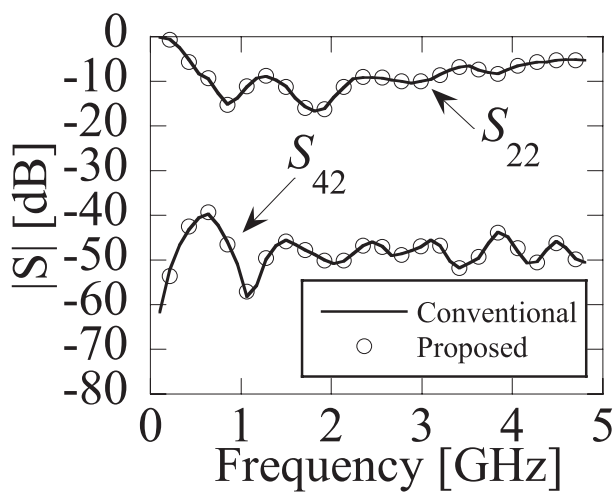

(b) $S_{22}, S_{42}$

Fig. 12 Comparison of simulated scattering parameters yielded by conventional method and proposed method using combined OFDM and $\mathrm{CI}$ technique $(D / L=2.0)$.

Table 2 Method comparison.

\begin{tabular}{|c|c|c|c|c|}
\hline Pulse & $\begin{array}{c}\text { Number of } \\
\text { antenna }\end{array}$ & $\begin{array}{c}\text { Accuracy of } \\
\text { analysis }\end{array}$ & $\begin{array}{c}\text { Inter-array } \\
\text { spacing }\end{array}$ & $\begin{array}{c}\text { Analysis } \\
\text { time }\end{array}$ \\
\hline Gaussian & 2 or $2 \times 2$ & Good & $\begin{array}{c}\text { Neighborhood } \\
\sim \text { Far }\end{array}$ & 1 \\
\hline OFDM & 2 & Fair & $\begin{array}{c}\text { Neighborhood } \\
\sim \text { Far }\end{array}$ & $\begin{array}{c}1 / 2 \\
1 / 4\end{array}$ \\
\hline CI & $2 \times 2$ & Fair & Far & $1 / 2$ \\
\hline OFDM-CI & $2 \times 2$ & Fair & Far & $1 / 4$ \\
\hline
\end{tabular}

nique exploits the temporal orthogonality and requires fast attenuation of the signal. As shown in Fig. 5, the RMS error increases if only one symbol is excited. Even when the CI technique is combined with the OFDM technique, a better result can be obtained because in CI technique concentrates the power of the signal into the beginning and middle of the symbol. Figure 12 shows the scattering parameters yield by the combined OFDM and CI technique; $D$ is set to $2.0 \mathrm{~L}$. The scattering parameters obtained by the proposed method agree very well with those of the conventional method. Additionally, the proposed method can reduce total computation time by $75 \%$ compared to the conventional method.

Finally, Table 2 shows a comparison of the conventional method and our proposed methods. These results con- firm that the proposed analysis methods can greatly reduce the computation time of multi-antenna simulations.

\section{Conclusion}

A fast FDTD analysis method for multi-antenna systems was proposed in this paper. This method exploits orthogonalities in both the frequency and time domains. Simulations showed that the proposed method outputs scattering parameters that agree well with those of the conventional method even though it is four times faster. The results shown in this paper prove that our proposed analysis method is effective in reducing the computation time of FDTD analysis of multi-antenna systems.

As a final remark, the proposed method can be applied to array antenna systems that have more than $2 \times 2$ antennas by using frequency orthogonality. But this will degrade the frequency resolution. This problem needs further investigation.

\section{Acknowledgements}

This research is partially supported by MEXT KAKENHI (22760261).

\section{References}

[1] G.J. Foschini and M.J. Gans, "Capacity when using diversity at transmit and receive sites and the Rayleigh-faded matrix channel is unknown at the transmitter," Proc. WINLAB Workshop on Wireless Information Network, March 1996.

[2] J.H. Winters, J. Salz, and R.D. Gitlin, "The impact of antenna diversity on the capacity of wireless communication systems," IEEE Trans. Commun., vol.42, no.2, pp.1740-1751, Feb. 1994.

[3] K.S. Yee, "Numerical solution of initial boundary value problems involving Maxwell's equations in isotropic media," IEEE Trans. Antennas Propag., vol.AP-14, no.4, pp.302-307, May 1966.

[4] K. Yokomakura, S. Sampei, H. Harada, and N. Morinaga, "A carrier interferometry based channel estimation technique for one-cell reuse MIMO-OFDM/TDMA cellular systems," Proc. IEEE VTC 2006Spring, vol.4, pp.1733-1737, May 2006.

[5] J.W. Wallace and M.A. Jansen, "The capacity of MIMO wireless systems with mutual coupling," Proc. IEEE 56th Veh. Technol. Conf., vol.2, pp.696-700, Sept. 2002.

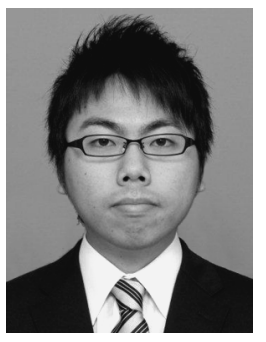

Mitsuharu Obara received B.E. degrees in Faculty of Electrical and Electronics Engineering from Iwate University in 2010. He is currently studying for M.E. degree at Iwate University. His research interests include FDTD analysis of multi-antenna systems. 


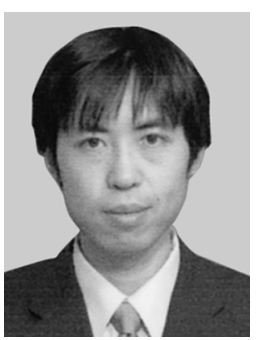

Naoki Honma received the B.E., M.E., and $\mathrm{Ph} . \mathrm{D}$. degrees in electrical engineering from Tohoku University, Sendai, Japan in 1996, 1998, and 2005, respectively. In 1998, he joined the NTT Radio Communication Systems Laboratories, Nippon Telegraph and Telephone Corporation (NTT), in Japan. He is now working for Iwate University. He received the Young Engineers Award from the IEICE of Japan in 2003, the APMC Best Paper Award in 2003, and the Best Paper Award of IEICE Communication Society in 2006, respectively. His current research interest is planar antennas for high-speed wireless communication systems. He is a member of IEEE.

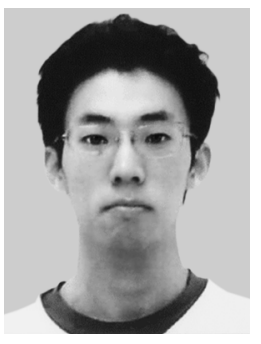

Yuto Suzuki received B.E. degree in Faculty of Electrical and Electronics Engineering from Iwate University in 2011. He is currently studying for M.E.degree at Iwate University. His research interests include beam switched antenna. 